Aim of the study: To investigate the effects of P27RF-Rho on hepatocellular carcinoma (HCC) cell growth and explore the possibility of using it as a novel therapeutic target for liver cancer treatment.

Material and methods: P27RF-Rho in HCC cells was silenced by lentivirus-mediated RNA interference, and the silencing effect was verified by RT$P C R$. Cell proliferation was determined by MTT and clone formation assay. Cell cycle phase and apoptosis were detected through FACS. The expression level of cell growth, apoptosis, and metastasis associated genes was detected by quantitative PCR.

Results: Lentivirus-mediated P27RFRho knockdown inhibited HCC cell growth and clone formation. P27RFRho silence induced cell cycle arrest and apoptosis. The mRNA level of genes associated with cell cycle, apoptosis, and invasion also significantly altered after P27RF-Rho knockdown. Cyclin A, CDK2, BCL-2, and MMP-9 were down-regulated. P27 and Bax were up-regulated.

Conclusions: P27RF-Rho knockdown inhibits HCC cell growth, and P27RFRho is probably a promising target for HCC treatment.

Key words: P27RF-Rho, hepatocellular carcinoma, RNA interference, cell growth.

Contemp Oncol (Pozn) 2017; 21 (1): 35-41 DOI: https://doi.org/10.5114/wo.2017.66654

\section{Lentivirus-mediated knockdown of P27RF-Rho inhibits hepatocellular carcinoma cell growth}

\author{
Shuli Xie ${ }^{1}$, Guangyi Wang ${ }^{1}$, Guofu Chen ${ }^{2}$, Mingguang Zhu ${ }^{3}$, Guoyue Lv ${ }^{1}$ \\ ${ }^{1}$ Department of General Surgery, The First Hospital of Jilin University, Jilin, China \\ 2Department of General Surgery, Daqing City People's Hospital, Daqing City, \\ Heilongjiang Province, China \\ ${ }^{3}$ Department of Immunology, School of Basic Medical Sciences, Jilin University, \\ Changchun, Jilin, China
}

\section{Introduction}

Hepatocellular carcinoma (HCC) is ranked as the third cause of cancer-related fatality worldwide. It was estimated that 748,300 new HCC cases and 695,900 fatalities occurred worldwide in 2008 [1]. Cancer is a systemic disease, and local invasion and distant metastasis are the main cause of death. Immortalised growth and infinite proliferation are the basic features of cancer. Rho-kinase plays a pivotal role in the organisation of cytoskeleton as well as in the regulation of transcription factors [2]. RhoC belongs to the Rho-kinase family. Its expression in normal tissue is very low or undetectable but is high in tumour. In studies about the relationship between RhoC and tumour, researchers are beginning to pay more attention to the regulation of RhoC activity. Existing data suggest that the regulation of RhoC activity plays an important role in cancer progression.

Deleted liver cancer 1 (DLC-1), a negative regulator of RhoA and RhoC pathway, has a strong inhibitory effect on cancer. DLC-1 was originally identified as a potential tumour suppressor in HCC. Its expression was lost or down-regulated in various cancers including liver, breast, lung, brain, stomach, colon, and prostate cancers due to either genomic deletion or aberrant DNA methylation [3]. In DLC-1 that lacks nasopharyngeal carcinoma cells, restoration of DLC-1 expression could attenuate cell proliferation, migration and clone formation in vitro, and reduce tumorigenesis potential in vivo [4]. Deletion or downregulated expression of DLC-1 is common in HCC. Restoration of DLC-1 expression in HCC cells resulted in caspase-3-mediated apoptosis, inhibition of cell growth, and invasiveness in vitro, as well as the inhibition of tumour formation in athymic nude mice [5].

Rho GDP dissociation inhibitory factor 2 (RhoGDI 2) has also attracted researchers' interest in recent years. Alterations in RhoGDI2 expression have been shown in a variety of human tumour types. It functions as a pro- or anti-tumorigenic and/or metastatic protein, varying greatly among cancer tissue types [6]. RhoGDI2 mRNA was significantly overexpressed in the HCC specimens compared with non-neoplastic liver specimens, and the RhoGDI2 mRNA and protein levels were higher in the HCC cell lines, especially the highly metastatic cell lines $97 \mathrm{~L}$ and $97 \mathrm{H}$ [7]. The clinical significance of RhoGDI2 in patients with colorectal carcinoma (CRC) was investigated. Over-expression of RhoGDI2 is associated with poor overall survival in CRC patients, especially those presenting in early stage. RhoGDI2 contributed to cell proliferation, motility, and invasion of CRC, at least in part, by activating the PI3K/Akt pathway [8]. However, low expression of RhoGDI2 is associated with poor outcome in bladder cancer patients. In animal models of human bladder cancer, high expression of RhoGDI2 suppressed lung metastasis by reducing the expression of proteoglycan versican [9]. 
P27RF-Rho is a newly discovered regulator of Rho GTPase activity. It combines with P27kip1, releasing the inhibition of P27kip1 to Rho protein, activating RhoA and RhoC [10]. However, the effects of P27RF-Rho on HCC cell proliferation, apoptosis, and metastasis have not yet been clarified by experiments. This experiment is designed to investigate the effects of P27RF-Rho on hepatocellular cell growth and to explore its possible mechanisms.

\section{Material and methods}

\section{Reagents and instruments}

Bel7402 (human hepatocellular carcinoma cell line) and HEK293T (Human Embryonic Kidney 293 cell line) cells were kept by the General Surgery Laboratory of the First Hospital, Jilin University. ShRNA vector U6-shRNACMV-copGFP-PGK-Puro was purchased from Huijun Biotechnological Company (China). Escherichia coli DH5a was purchased from Invitrogen. Restriction enzymes and DNA ligase were from Thermo Fisher Scientific Company (USA). DNA gel recovery kit and kit for rapid extraction of plasmid DNA were purchased from Promega Corporation (USA). Kits for quantitative PCR and RT-PCR, as well as DNA Marker DL2000, were purchased from Takara Company (China). Lipofectamine 2000 and Annexin V-FITC apoptosis detection kit were from Invitrogen (USA). Real-time PCR System (Stratagene M×3005p) was purchased from Agilent Technologies Co. Ltd (USA). Flow Cytometry was purchased from BD FACSCalibur (BD, USA). All antibodies were purchased from Santa Cruz (USA). All enzymes were purchased from Takara.

\section{Construction of shRNA vectors}

Two shRNA vectors, one for P27RF-Rho knockdown and another for negative control, were designed and constructed. The RNA interference sequence targeting P27RF-Rho was 5 '- GGAGCTGGTTGTACAGTTTTCAAGAGAAACTGTACAACCAGCTCC TTTTT - 3'(sense), and 5 '- AAAAAGGAGCTGGTTGTACAGTTTCTCTTGAAAACTGTACAACCAGCTCC GTAC - 3'(antisense). Negative control (scramble) was 5 '- GTTGCATACGTGCGGTGATAT TCAAGAGATATCACCGCACGTATGCAAC TTTTT - 3'(sense) and 5'- AAAAAGTTGCATACGTGCGGTGATATCTCTTGAATATCACCGCACGTATGCAAC GTAC - 3'(antisense). The shRNA vector was purchased from Huijun Biotechnology Company (China), and the oligo DNA fragments were synthesised by this company. Double-stranded DNA was obtained by oligo DNA annealing. In brief, sense and antisense oligonucleotides (100 pmol) $2 \mu \mathrm{l}$ were mixed with $10 \times$ annealing buffer $2 \mu \mathrm{l}$ and $\mathrm{dd}_{2} \mathrm{O} 14 \mu \mathrm{l}$ in an Eppendorf tube, with a total reaction volume of $20 \mu \mathrm{l}$. The annealing procedure began at $94^{\circ} \mathrm{C}$ for three minutes, then the temperature was lowered with a gradient of $5^{\circ} \mathrm{C}$, and kept at each temperature gradient for 5 minutes, until room temperature was reached. After enzyme digestion with Kpn and HPal. ShRNA vector and the double stranded DNA were ligated with T4 DNA ligase. The ligation mixture was incubated at $16^{\circ} \mathrm{C}$ overnight. It was transformed into Escherichia coli DH5a. After transformation, positive clones were identified by PCR and sequenced. The two shRNA vectors were named U6-shRNA-
CMV-copGFP-PGK-Puro-P27RF-Rho (P27RF-Rho-siRNA for short) and U6-shRNA-CMV-copGFP-PGK-Puro-Scramble (P27RF-Rho-Scramble for short).

\section{Lentivirus packaging and cell infection}

$1.2 \times 10^{7}$ HEK 293 T cells were inoculated in a plate $60 \mathrm{~mm}$ in diameter, cultivated at $37^{\circ} \mathrm{C}$ in a humidified incubator containing $5 \% \mathrm{CO}_{2}$, and left to grow overnight. The cells were transfected with a mixture of shRNA vector $4.0 \mu \mathrm{g}$, psPAX2 vector $2.0 \mu \mathrm{g}$, and pMD2G vector $2.0 \mu \mathrm{g}$, using Lipofectamine 2000 according to the manufacturer's instructions. Transfection efficiency was determined by fluorescence microscope. Briefly, three non-overlapping vision fields were randomly selected, the number of positive cells out of every 100 cells in total was determined, and the corresponding transfection efficiency was then calculated according to the following formula: transfection efficiency $=$ total number of positive cells in THREE vision fields/ $300 \times 100 \%$. The transfection efficiency above $90 \%$ was considered as a successful transfection. Supernatant containing lentivirus was harvested 48 and 72 hours after transfection, respectively, using Lenti-Pac ${ }^{\mathrm{TM}}$ Lentivirus Concentration Solution (GeneCopoeia,USA), according to the manufacturer's instructions. Cells were infected with the lentiviruses at Multiplicity of Infection (MOI) of 20. Forty-eight hours after lentivirus infection, puromycin at the concentration of $5 \mu \mathrm{g} / \mathrm{ml}$ was applied to screen stable infected cell lines. Transfection efficiency exceeded $95 \%$ in the screened cell lines.

\section{RT-PCR detection of P27RF-Rho silencing}

PP27RF-Rho knockdown was verified by RT-PCR. Briefly, total RNA was extracted according to TRIzol kit (Tiangen, China) instruction, and cDNA was synthesised from the extracted total RNA using a RT-PCR kit (TAKARA, China) following the manufacturer's instructions. The specific primers used for PCR were as follows: P27RF-Rho sense 5'- TGTGACCGGAAGGGCTCCT-3' and antisense 5'-GAGGAGAAGAGCTGTCCAAG-3'(580 base pair). $\alpha$-tubulin was used as an internal reference, upstream primer 5'- CACCCGTCTTCAGGGCTTCTTGGTTT-3', downstream primer 5'- CATTTCACCATCTGGTTGGCTGGCTC-3'(527 base pair). All specific primers were chemically synthesised (Sangon Biotech, China). The RT-PCR reactions were performed using the PCR Thermal Cycler Dice (TAKARA, China). The amplification conditions for P27RF-Rho were as follows: $94^{\circ} \mathrm{C}$ for two minutes followed by 35 cycles at $94^{\circ} \mathrm{C}$ for 30 seconds, $57^{\circ} \mathrm{C}$ for 30 seconds, and $72^{\circ} \mathrm{C}$ for 60 seconds, and a final extension at $72^{\circ} \mathrm{C}$ for 5 minutes. The PCR conditions for $\beta$-actin were as follows: $94^{\circ} \mathrm{C}$ for two minutes followed by 25 cycles at $94^{\circ} \mathrm{C}$ for 30 seconds, $56^{\circ} \mathrm{C}$ for 30 seconds, and $72^{\circ} \mathrm{C}$ for 60 seconds, and a final extension at $72^{\circ} \mathrm{C}$ for 5 minutes. The amplified RT-PCR products were separated on $1.5 \%(\mathrm{~W} / \mathrm{V})$ agarose containing ethidium bromide (both from Sangon Biotech) for 30 minutes. The results of electrophoresis were photographed, using the Gel Imaging System. The signal densities of the gels were analysed using software affiliated to the machine (Bio-Rad, USA). 


\section{Cell growth assay by MTT}

Briefly, exponentially growing cells were inoculated into 96-well plates with $0.5 \times 10^{4}$ cells per well, culturing in incubator at $37^{\circ} \mathrm{C}$ in a humidified atmosphere of $5 \% \mathrm{CO}_{2}$. After incubation for 24, 48, 72, 96, 120, and 144 hours, $10 \mathrm{ml}$ of sterile MTT $(5 \mathrm{mg} / \mathrm{ml})$ was added to each well. Following incubation at $37^{\circ} \mathrm{C}$ for four hours, the reaction was blocked by adding $100 \mathrm{ml}$ of dimethyl sulfoxide. The formazan production was determined by measurement of the spectrometric absorbance at $570 \mathrm{~nm}$. The values obtained are proportional to the amount of viable cells, and each experiment was repeated three times.

\section{Clone formation assay}

Cells were seeded in 6 -well plates with $5 \times 10^{2}$ cells per well and cultured at $37^{\circ} \mathrm{C}$ with $5 \% \mathrm{CO}_{2}$ for 14 days. The cell colonies were washed twice with PBS, fixed in $4 \%$ paraformaldehyde for 30 minutes, and stained with Giemsa for 20 minutes. Individual colonies with more than 50 cells were counted under a microscope. Clone formation ratio $(\%)=$ clone number/planted cell number by $100 \%$.

\section{Cell cycle detection by flow cytometry}

Exponentially growing cells in each group were seeded in six-well plates. After 48 hours, cells were harvested and washed with pre-chilled PBS, and fixed with pre-chilled $70 \%$ ethanol at $4^{\circ} \mathrm{C}$ overnight. The fixed cells were washed with $1 \mathrm{ml}$ PBS, adding $500 \mu \mathrm{l}$ PBS containing $50 \mu \mathrm{g} / \mathrm{ml}$ ethidium bromide (propidium iodide, PI), $100 \mathrm{\mu g} / \mathrm{ml}$ RNase A, $0.2 \%$ Triton $X-100$, and incubated at $4^{\circ} \mathrm{C}$ for 30 minutes in the dark. About 20,000-30,000 cells were counted through flow cytometry, detecting red fluorescence at excitation wavelength $488 \mathrm{~nm}$, and light scattering was detected as well.

\section{Cell apoptosis detection by flow cytometry}

Exponentially growing cells in each group were seeded in 6-well plates. After 48 hours, cells were harvested and washed twice with pre-chilled PBS (centrifugation at 2000 rpm, 5 minutes). About $5 \times 10^{5}$ cells were collected. In apoptosis analysis, a Dead Cell Apoptosis Kit with annexin $\checkmark$ and PI for Flow Cytometry (Invirogen, USA) was used according to the manufacturer's instructions. Briefly, after PBS washing, cells were re-suspended in $1 \times$ annexing binding buffer at a density of $1 \times 10^{6}$ cells $/ \mathrm{ml}$. Then, $5 \mu \mathrm{l}$ of FITC Annexin- $V$ and $1 \mu \mathrm{l} 100 \mu \mathrm{g} / \mathrm{ml} \mathrm{Pl}$ were added to each $100 \mu \mathrm{l}$ of the cell suspension and the samples were incubated at room temperature for 15 minutes in the dark. After incubation, $400 \mu \mathrm{l} 1 \times$ annexin binding buffer was added, mixed gently, and the samples were kept on ice. Apoptosis was analysed by FACSCalibur using Cell-Quest software (BD, USA). The cells undergoing apoptosis were Annexin-V-FITC-positive and $\mathrm{PI}$-negative. All experiments were repeated three times.

Cell growth, metastasis, and apoptosis-related gene expression determination by real-time PCR

In brief, total RNA was extracted using TRIzol reagent (Tiangen, China).
The reverse transcription reactions were carried out following the kit manufacturer's instructions. Real-time quantitative PCR analysis was performed in Real-time PCR System (Stratagene M×3005p, USA), using SYBR Master Mixture (TaKaRa, Japan). The primer sequences for PCR amplification of cyclin A were 5'- ATTGGTCCCTCTTGATTA -3'(sense) and 5'-ATGTCTGGCTGTTTCTTC -3'(antisense). CDK2 were 5'-GGCCTAGCTTTCTGCCATTC-3' (sense) and 5'-CCCAGGAGGATTTCAGGAGC-3'(antisense). P27 were 5'- AATAAGGAAGCGACCTGCAA-3'(sense) and 5'- CTCCACAGAACCGGCATT -3'(antisense). MMP-9 were 5'-AGTGGGCTACGTGACCTATGA- 3'(sense) and 5'-CGGCAAACTGGCTCCTT- 3'(antisense). BCl-2 were 5'- GAGGATTGTGGCCTTCTTTG-3'(sense) and 5'- GTGCCGGTTCAGGTACTCA -3'(antisense). BAX were 5'- CCGATTCATCTACCCTGCTG -3'(sense) and 5'- TGAGCAATTCCAGAGGCAGT -3'(antisense). GAPDH were 5'-AACGGATTTGGTCGTATTG-3'(sense) and 5'-CTGGAAGATGGTGATGGG-3'(antisense). GAPDH was applied as an internal control. Results were normalised to GAPDH expression. Then, the differential expression of these genes was analysed by the $\Delta C T$ method and expressed as the fold changes. All experiments were repeated three times.

\section{Statistical analysis}

All results were reported as means \pm SD. Statistical significance was evaluated by one-way analysis of variance using SPSS software (Version 10.0; SPSS). Values of $p<0.05$ were considered statistically significant.

\section{Results}

\section{Cell growth was inhibited by P27RF-Rho} knockdown

The level of P27RF-Rho mRNA was significantly lower in the P27RF-Rho-siRNA group than in the parental Bel7402 and negative control groups ( $0.21 \pm 0.07$ vs. $0.83 \pm 0.10,0.88$ $\pm 0.12, p<0.01$ ). From the second day on, cell growth in the P27RF-Rho-siRNA group was remarkably slower than in the parental Bel7402 and negative control groups (0.44 \pm 0.01 vs. $0.62 \pm 0.03$ and $0.60 \pm 0.04, p<0.01$ ). The clone formation ratio of cells in the P27RF-Rho-siRNA group was significantly lower than in the parental Bel7402 and negative control groups ( $13.50 \pm 1.73 \%$ vs. $49.83 \pm 6.43 \%$ and $47.17 \pm 4.04 \%, p<0.01$ ), suggesting that P27RF-Rho-siRNA inhibited cell clone formation (Fig. 1).

\section{P27RF-Rho silencing resulted in cells stagnating in} quiescent phase and apoptosis

In the P27RF-Rho-siRNA group, cells in the G0/G1 phase were significantly more than in the parental Bel7402 and negative control groups $(70.70 \pm 20.23 \%$ vs. $59.43 \pm 1.80 \%$ and $61.81 \pm 2.67 \%, p<0.01)$. Cells in the G2/M phase in the P27RF-Rho-siRNA group were noticeably less than in the parental Bel7402 and negative control groups (2.78 $\pm 0.98 \%$ vs. $12.59 \pm 0.74 \%$ and $12.58 \pm 1.02 \%, p<0.01$ ), suggesting that cell growth was inhibited after P27RF-Rho knockdown. More apoptosis occurred in the P27RF-Rho knockdown group than in the parental Bel7402 and negative control groups (45.86 $\pm 2.71 \%$ vs. $8.55 \pm 0.90 \%$ and 

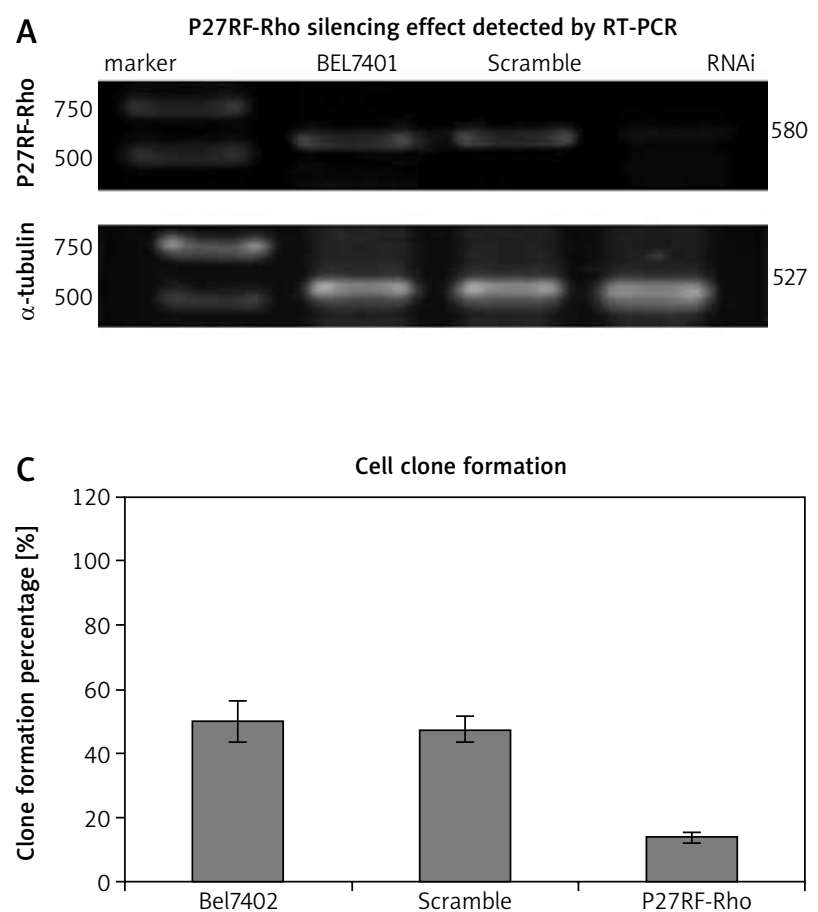

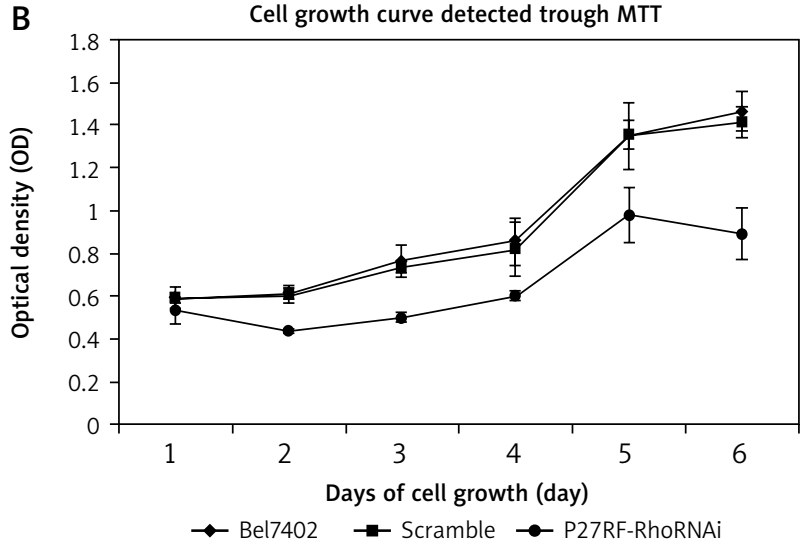

Fig. 1. Cell growth after P27Rf-Rho knockdown. A) P27Rf-Rho was knocked down by RNA interference. B) Cell growth was depressed after P27Rf-Rho knockdown. C) Cell clone formation inhibited by P27Rf-Rho silence

$7.87 \pm 1.21 \%, p<0.01)$. No significant difference was found in $\mathrm{S}$ phase among the three groups (Fig. 2).

Cell growth, apoptosis, and metastasis-associated genes changed after P27RF-Rho knockdown

Cell growth, apoptosis, and metastasis-associated genes were determined by quantitative PCR (Fig. 3). The results showed that the following mRNA levels were significantly lower in the P27RF-Rho-siRNA group than in the parental Bel7402 and negative control groups: cyclin A $(0.38 \pm 0.06$ vs. $2.75 \pm 0.48$ and $2.76 \pm 0.42, p<0.01)$, CDK2 (0.59 \pm 0.16 vs. 4.19 \pm 0.48 and $4.09 \pm 0.52, p<0.01)$, MMP-9 (0.35 \pm 0.08 vs. 3.13 \pm 0.36 and $3.14 \pm 0.58, p<0.01)$, and $B C L-2(0.37 \pm 0.03$ vs. 2.64 \pm 0.56 and $3.03 \pm 0.43, p<0.01)$. The following mRNA levels in the P27RF-Rho-siRNA group were notably increased when compared with the parental Bel7402 and negative control groups: P27 (1.13 \pm 0.13 vs. $0.19 \pm 0.04$ and $0.18 \pm 0.06, p<0.01)$ and BAX (1.04 \pm 0.09 vs. $0.12 \pm 0.03$ and $0.11 \pm 0.03, p<0.01)$.

\section{Discussion}

Hepatocellular carcinoma has an insidious onset. Most patients are in a late tumour stage when they go to hospital for diagnosis, thus losing operation opportunity. Unfortunately, there is no effective drug for HCC at present. Therefore, the therapeutic target for HCC is a hot point in liver cancer research.

The vast majority of HCC patients have the experience of suffering chronic liver diseases, which leads to a series of genetic events, resulting in liver cell malignant transformation [11]. HCC is the result of accumulation of genetic mutations. Rho GTPases play an important role in HCC. P27RF-Rho is a newly discovered protein regulating Rho
(RhoA and RhoC) activity [12]. Rapid growth and unlimited proliferation are the basic characteristics of malignant cells. This experiment found that lentivirus-mediated P27RF-Rho knockdown led to decreased HCC cell growth and increased cell apoptosis. These results suggested that P27RF-Rho was an important regulator of HCC cell growth.

Cell growth is decided by a series of cell-cycle regulatory proteins. Rho activation can result in a variety of alterations of these proteins and ultimately lead to malignant transformation. Mouse embryo fibroblasts with DLC-1 knockout underwent neoplastic transformation. In addition to inactivation of DLC-1 and increased activity of Rho and $\mathrm{Cdc42}$, the neoplastic transformation depended on the subsequent decreased expression of P15 and $P 16$, together with increased expression and activation of CDK4/6 [13]. In this experiment, we found that cyclin A and CDK2 expression were depressed, and P27 expression was increased after P27RF-Rho knockdown. Cyclin A promoted cells passing through checkpoint G1 to G2 phase [14]. Cyclin A decrement blocked HCC cell growing from G1 to G2 phase, making G1 phase cells increase and S phase cells decrease. CDK2 could directly affect cell cycle, determining apoptosis and tumour development [15]. P27 worked as a tumour suppressing protein, inhibiting the function of CDK complex, regulating cell progression from $\mathrm{G} 1$ to $\mathrm{S}$ phase [16]. In this experiment, it was highly possible that P27RF-Rho inhibited HCC cell proliferation by affecting cyclin A, CDK2, and P27 expression. The mechanisms of this inhibition need to be further clarified.

Tumour metastasis involves tumour cell infiltration and extracellular matrix (ECM) degradation. Matrix metalloproteinases (MMPs) are a collection of enzymes that 
A

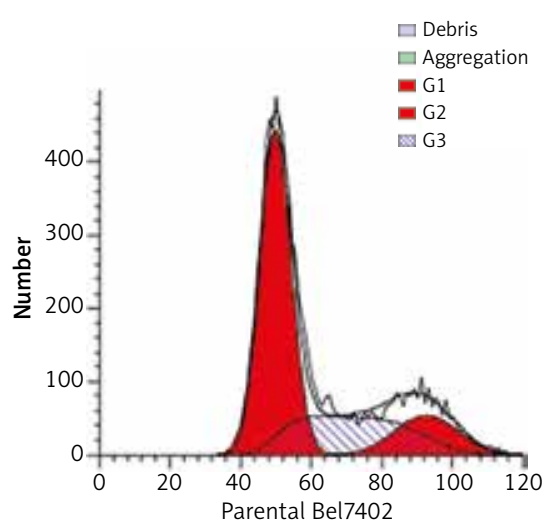

Cell cycle changes detected by FACS
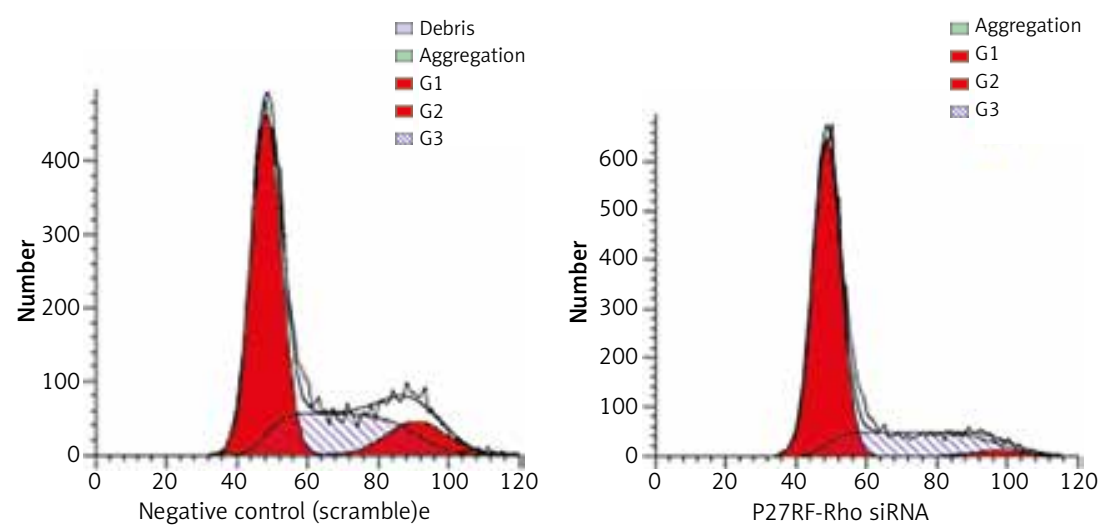

B

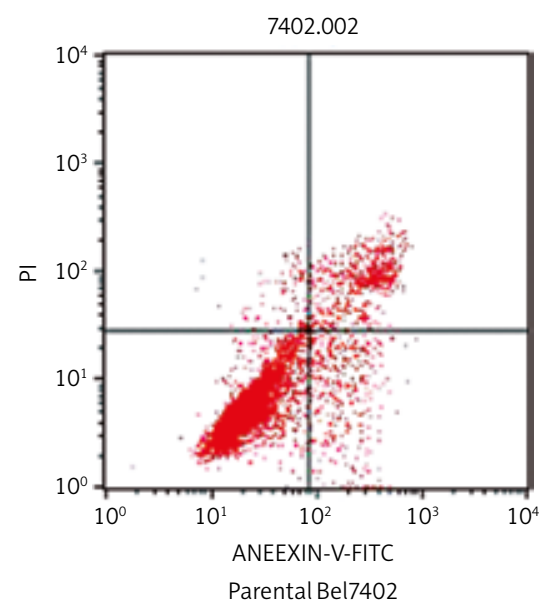

Cell cycle changes detected by FACS

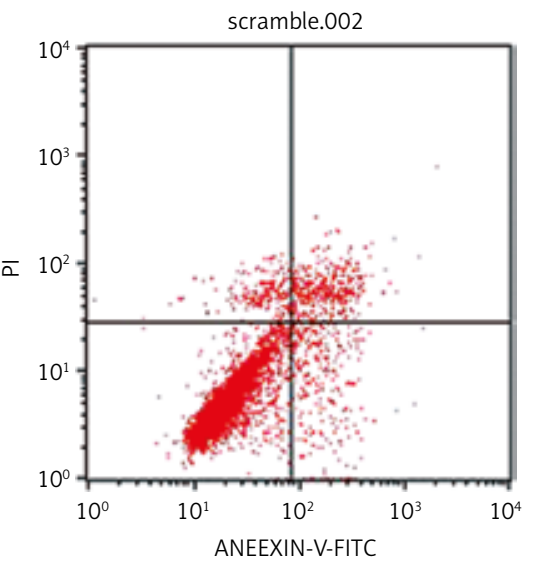

Negative control (scramble)e

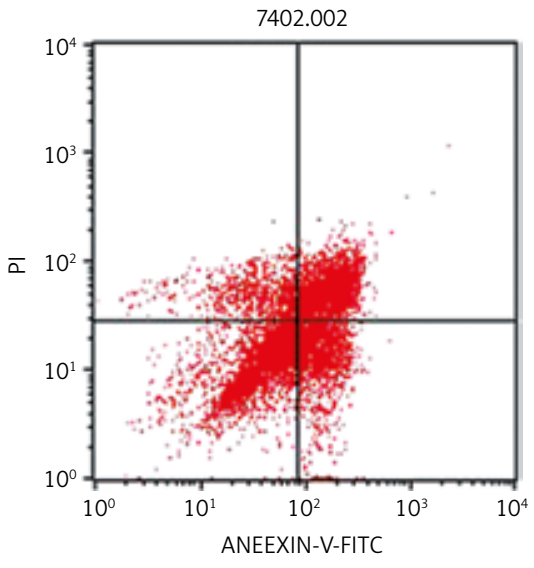

P27RF-Rho-siRNA

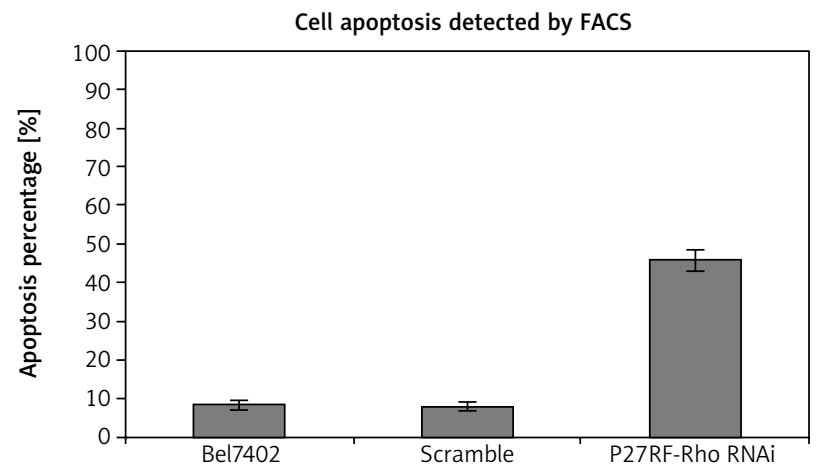

Fig. 2. Cell cycle and apoptosis in different groups determined by FACS. A) More cells arrested in G0/G1 phase and less cells stayed in G2/M phase in P27RF-Rho knockdown group. B) P27RF-Rho knockdown led to more cell apoptosis 

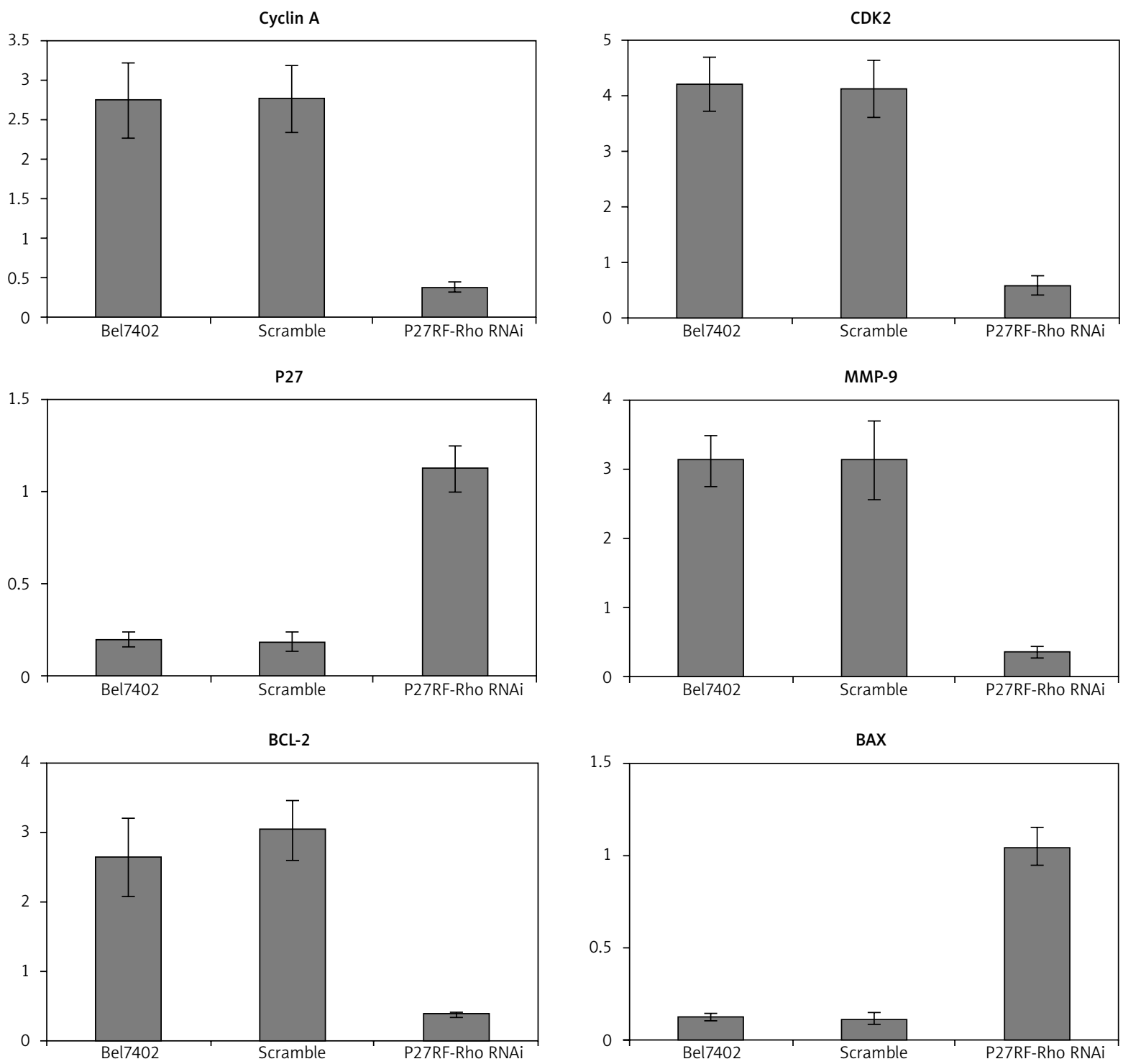

Fig. 3. The mRNA level of genes associated with cell cycle and apoptosis detected by qPCR. P27RF-Rho knockdown led to less expression of cyclin A, CDK2, MMP-9 and Bcl-2 mRNA. P27 and Bax mRNA levels increased after P27RF-Rho silencing

breakdown ECM and basement membrane proteins. MMP-9 could degrade gelatine, a layer adhesion protein, correlating with tumour invasion and metastasis. MMP-9 was over-expressed in a variety of malignant neoplasms. Inhibition of MMP-9 expression or its activity could block tumour cell invasion and migration [17]. RhoGDI2-mediated cellular invasion required the PI3K/Akt signalling-dependent expression of MMPs [5]. In this experiment, we found that MMP-9 expression was decreased after P27RFRho knockout, suggesting the attenuation of HCC cell invasion and metastasis. The mechanisms underlying this correlation between MMP-9 and P27RF-Rho are not available.

Apoptosis is a biological process, in which multicellular organisms remove undesired or abnormal cells. It is very important for internal environment stability and organ development. In the pathological process of tumours, apoptosis disorder occurs. The BCL-2 family is the key component of cell apoptosis regulation. It consists of three family members, including BCL-2, BAX, and $\mathrm{BH}$.
BCL-2 inhibited cell apoptosis by inhibiting asparagi protease (Caspase), while BAX and $\mathrm{BH} 3$ promoted cell apoptosis [18]. Tumour cells might use some of the molecular mechanisms to suppress apoptosis and acquire resistance to apoptotic reagents, for example, by the expression of antiapoptotic proteins such as $\mathrm{Bcl}-2$ or by the downregulation of proapoptotic proteins such as BAX [18]. We found decreased BCL-2 expression and increased BAX expression after P27RF-Rho silence in this experiment, suggesting that P27RF-Rho might promote cell survival and inhibited apoptosis by regulating BCL-2 and BAX expression.

Lentivirus is a promising carrier for tumour gene therapy. It can deliver siRNA targeting specific oncogenes to tumour cells. Many studies used adenovirus or lentivirus vectors to deliver siRNA into tumour cells in vitro and/or in vivo [19]. In this experiment, we constructed a retrovirus-mediated siRNA vector targeting P27RF-Rho and transfected it into HCC cells in vitro, resulting in depressed cell growth and enhanced apoptosis, demonstrating the possi- 
bility to use this method as a therapy for HCC. SiRNA can be obtained through chemical synthesis, and lentivirus can work as a safe and simple carrier for gene silence therapy. The high transfection efficiency can meet the need for gene silencing.

In summary, we found that in this experiment P27RFRho knockdown in HCC cells resulted in inhibited cell growth and increased cell apoptosis. The alterations of cell growth, apoptosis, and metastasis might be controlled by the associated proteins such as cyclin A, cyclin G, CDK2, P27, MMP-2, BCL-2, and BAX. Our findings suggest that P27RF-Rho is probably a promising novel target for HCC treatment, and lentivirus-mediated P27RF-Rho knockdown might be used as an effective biological therapy to deal with HCC. These results should be further confirmed in vivo.

The authors declare no conflict of interest.

\section{References}

1. Jemal A, Bray F, Center MM, Ferlay J, Ward E, Forman D. Global cancer statistics. CA Cancer J Clin 2011; 61: 69-90.

2. Amin E, Dubey BN, Zhang SC, et al. Rho-kinase: regulation, (dys) function, and inhibition. Biol Chem 2013; 394: 1399-410.

3. Liao YC, Lo SH. Deleted in liver cancer-1 (DLC-1): a tumor suppres sor not just for liver. Int J Biochem Cell Biol 2008; 40: 843-47.

4. Feng X, Li C, Liu W, et al. DLC-1, a candidate tumor suppressor gene, inhibits the proliferation, migration and tumorigenicity of human nasopharyngeal carcinoma cells. Int J Oncol 2013; 42: 1973-84.

5. Zhou X, Thorgeirsson SS, Popescu NC. Restoration of DLC-1 gene expression induces apoptosis and inhibits both cell growth and tumorigenicity in human hepatocellular carcinoma cells. Oncogene 2004; 23: 1308-13.

6. Griner EM, Theodorescu D. The faces and friends of RhoGDI2. Cancer Metastasis Rev 2012; 31: 519-28.

7. Fang Y, Yi J, Lizhi L, Qiucheng C. Rho GDP dissociation inhibitor beta promotes cell proliferation and invasion by modulating the AKT pathway in hepatocellular carcinoma. DNA Cell Biol 2014; 33: 781-6.

8. Li X, Wang J, Zhang X, Zeng Y, Liang L, Ding Y. Overexpression of RhoGDI2 correlates with tumor progression and poor prognosis in colorectal carcinoma. Ann Surg Oncol 2012; 19: 145-53.

9. Said N, Sanchez-Carbayo M, Smith SC, Theodorescu D. RhoGDI2 suppresses lung metastasis in mice by reducing tumor versican expression and macrophage infiltration. J Clin Invest 2012; 122: 1503-18.

10. Nada S, Mori S, Takahashi Y, Okada M. p18/LAMTOR1: A late Endosome/lysosome-specific anchor protein for the mTORC1/MAPK signaling pathway. Methods Enzymol 2014; 535: 249-63.

11. Grise F, Bidaud A, Moreau V. Rho GTPases in hepatocellular carcinoma. Biochim Biophys Acta 2009; 1795: 137-51.

12. Hoshino D, Koshikawa N, Seiki M. A p27kip1-binding protein, p27RF-Rho, promotes cancer metastasis via activation of RhoA and RhoC. J Biol Chem 2011; 286: 3139-48.

13. Qian X, Durkin ME, Wang D, et al. Inactivation of the Dlc1 gene cooperates with downregulation of p15INK4b and p16Ink4a, leading to neoplastic transformation and poor prognosis in human cancer. Cancer Res 2012; 72: 5900-11.

14. van Zon W, Wolthuis RM. Cyclin A and Nek2A: APC/C-Cdc20 substrates invisible to the mitotic spindle checkpoint. Biochem Soc Trans 2010; 38: 72-7.

15. Asghar U, Witkiewicz AK, Turner NC, Knudsen ES. The history and future of targeting cyclin-dependent kinases in cancer therapy. Nat Rev Drug Discov 2015; 14: 130-46.
16. Roy A, Banerjee S. p27 and leukemia: cell cycle and beyond. J Cell Physiol 2015; 230: 504-9.

17. Farina AR, Mackay AR. Gelatinase B/MMP-9 in tumour pathogenesis and progression. Cancers (Basel) 2014; 6: 240-96.

18. Wong WW, Puthalakath $\mathrm{H}$. BCl-2 family proteins: the sentinels of the mitochondrial apoptosis pathway. IUBMB Life 2008; 60: 390-7.

19. Prados J, Melguizo C, Roldan H, Alvarez PJ, Ortiz R, Arias JL, Aranega A. RNA interference in the treatment of colon cancer. Bio Drugs 2013; 27: 317-27.

\section{Address for correspondence}

\section{Guoyue Lv}

Department of General Surgery

The First Hospital of Jilin University, Changchun, Jilin

130021 Changchun, China

e-mail: xieshuli6310@hotmail.com

Submitted: 28.10 .2015

Accepted: 22.06 .2016 